\title{
On shock waves and the role of hyperthermal chemistry in the early diffusion of overdense meteor trains
}

\author{
Elizabeth A. Silber, ${ }^{1 *}$ Wayne K. Hocking, ${ }^{2}$ Mihai L. Niculescu, ${ }^{3}$ Maria Gritsevich ${ }^{4,5,6}$ \\ and Reynold E. Silber ${ }^{7}$ \\ ${ }^{1}$ Department of Earth, Environmental and Planetary Science, Brown University, Providence, RI 02912, USA \\ ${ }^{2}$ Department of Physics and Astronomy, University of Western Ontario, London, ON N6A 3K7 Canada \\ ${ }^{3}$ INCAS - National Institute for Aerospace Research 'ElieCarafoli', Flow Physics Department, Numerical Simulation Unit, Bucharest 061126, Romania \\ ${ }^{4}$ Department of Physics, University of Helsinki, Gustaf Hällströminkatu 2a, P.O. Box 64, FI-00014 Helsinki, Finland \\ ${ }^{5}$ Department of Computational Physics, Dorodnicyn Computing Centre, Federal Research Center 'Computer Science and Control' of the Russian Academy of \\ Sciences, Vavilova St 40, 119333 Moscow, Russia \\ ${ }^{6}$ Institute of Physics and Technology, Ural Federal University, 620002 Ekaterinburg, Russia \\ ${ }^{7}$ Department of Earth Sciences, University of Western Ontario, London, ON N6A 3B7, Canada
}

Accepted 2017 April 12. Received 2017 April 5; in original form 2017 February 7

\begin{abstract}
Studies of meteor trails have until now been limited to relatively simple models, with the trail often being treated as a conducting cylinder, and the head (if considered at all) treated as a ball of ionized gas. In this article, we bring the experience gleaned from other fields to the domain of meteor studies, and adapt this prior knowledge to give a much clearer view of the microscale physics and chemistry involved in meteor-trail formation, with particular emphasis on the first 100 or so milliseconds of the trail formation. We discuss and examine the combined physicochemical effects of meteor-generated and ablationally amplified cylindrical shock waves that appear in the ambient atmosphere immediately surrounding the meteor train, as well as the associated hyperthermal chemistry on the boundaries of the high temperature post-adiabatically expanding meteor train. We demonstrate that the cylindrical shock waves produced by overdense meteors are sufficiently strong to dissociate molecules in the ambient atmosphere when it is heated to temperatures in the vicinity of $6000 \mathrm{~K}$, which substantially alters the considerations of the chemical processes in and around the meteor train. We demonstrate that some ambient $\mathrm{O}_{2}$, along with $\mathrm{O}_{2}$ that comes from the shock dissociation of $\mathrm{O}_{3}$, survives the passage of the cylindrical shock wave, and these constituents react thermally with meteor metal ions, thereby subsequently removing electrons from the overdense meteor train boundary through fast, temperature-independent, dissociative recombination governed by the second Damköhler number. Possible implications for trail diffusion and lifetimes are discussed.
\end{abstract}

Key words: shock waves - Earth - meteorites - meteors - meteoroids.

\section{INTRODUCTION}

The physics of meteoric phenomena can be divided into three basic components (Dressler 2001). The first two, the dynamics of the meteoroid motion in the atmosphere (e.g. Boyd 2000; Gritsevich 2009), and aspects of the chemical and plasma kinetics of thermalized atoms and molecules deposited in the ambient atmosphere by meteor ablation (e.g. Plane 2012; Plane, Feng \& Dawkins 2015) have been subjects of numerous studies. The third component, which has not received sufficient attention to date, is concerned with cause and effects of meteor-generated shock waves and the closely related small-scale physical and chemical processes occurring in, and on the boundary of, the extreme environment of the high-temperature adiabatically formed meteor trail in the initial stages of the expansion. This aspect of the physicochemical evolution of overdense meteor trails (defined shortly) is the focus of this study.

Consequently, the broad aim of this work is to present an overview and examine the role of frequently neglected meteor cylindrical shock waves and the associated hyperthermal chemistry. We 


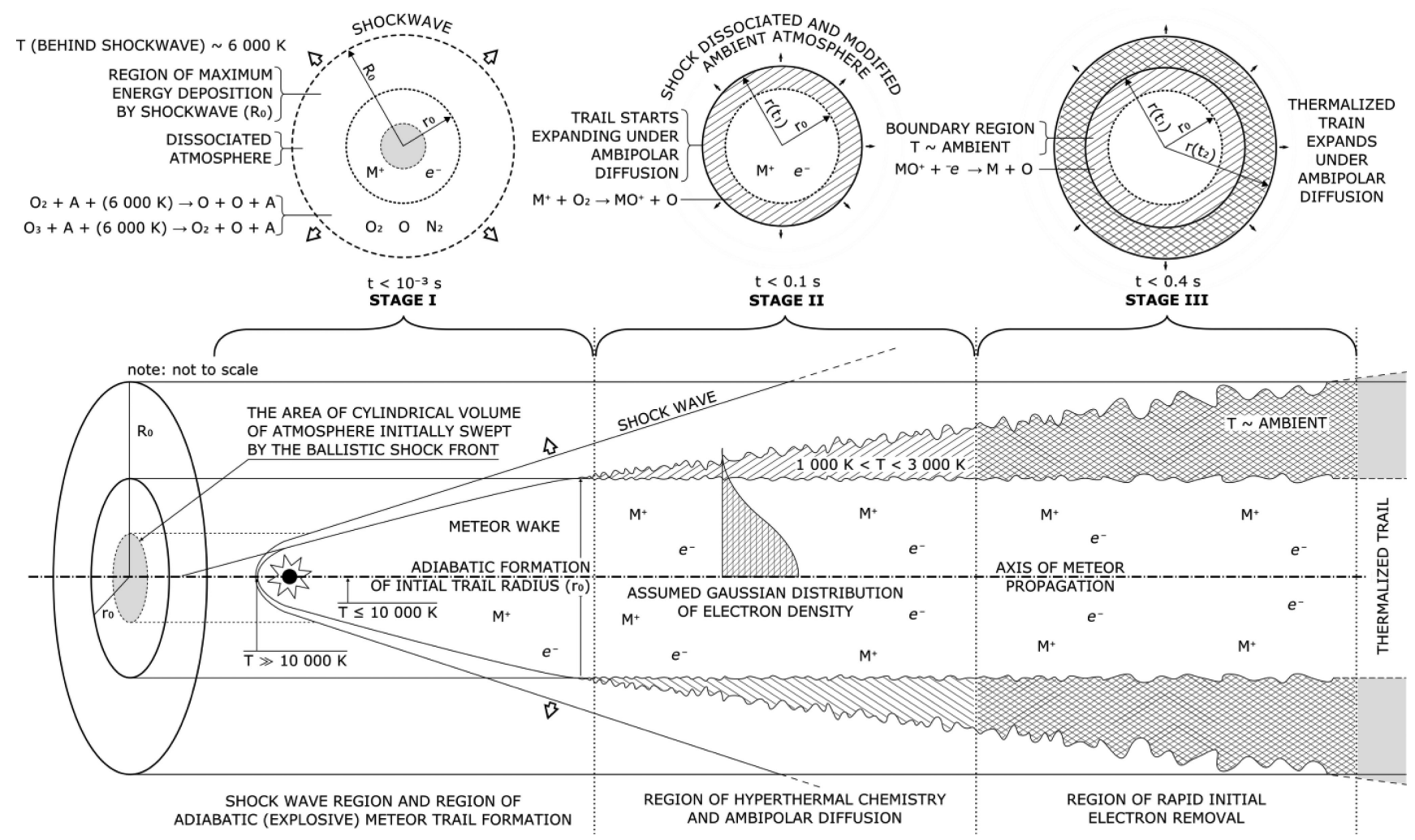

Figure 1. Schematic depiction of an overdense meteor's early evolution, in which three distinct stages can be recognized. In the first stage, the ablating meteoroid with the shock front in front sweeps the cylindrical volume of ambient atmosphere (depicted by the small grey circle), ionizing and dissociating atmospheric gasses. This stage also coincides with the cylindrical shock wave expanding radially outward, perpendicular to the meteor axis of propagation, with enough energy deposited within $R_{0}$ to dissociate $\mathrm{O}_{2}$ and $\mathrm{O}_{3}$ in the ambient atmosphere, but not enough for $\mathrm{N}_{2}$ dissociation (see the main text for discussion). In stage two, the adiabatically formed meteor train (which can be approximated as quasi-neutral plasma with the Gaussian radial electron distribution), begins to expand under ambipolar diffusion and thermalizes. This stage coincides with formation of metal ion oxides that takes place and is appreciable between approximately $(3000-1500 \mathrm{~K})$ at the boundary region of the diffusing trail. In this reaction, an ablated meteoric metal ion will react in a thermally driven reaction with the shock-dissociated product of ozone $\left(\mathrm{O}_{2}\right.$ in ground and excited states). In the stage three, in the almost thermalized train, the newly formed metal ion oxide will consume electrons rapidly by temperature-independent dissociative recombination (see the main text for discussion).

discuss the nature of physicochemical and associated processes behind the potentially rapid and short-lasting electron removal from post-adiabatically expanding high-temperature overdense meteor train boundaries. As the role of meteor-produced shock waves and hyperthermal chemistry phenomena associated with larger meteors has not been covered to a significant degree in the literature, except in a very few selected works (e.g. Menees \& Park 1976; Park \& Menees 1978; Berezhnoy \& Borovička 2010), we readdress that issue here. Hence, in this paper, we also present an extended discussion of the relevant aspects of shock waves, hydrodynamic phenomena and hyperthermal chemistry, as they may pertain to the topic of early diffusion of overdense meteors.

This paper is organized as follows. In Section 2, we provide the fundamentals and background pertaining to the evolution and behaviour of overdense meteor trains; in Section 3, we discuss the hyperthermal chemistry, while in Section 4, we consider the dynamic and physicochemical effects of overdense meteor cylindrical shock waves, including a computational model; and finally, our conclusions are presented in Section 5. Our computational model of meteor atmospheric entry at $80 \mathrm{~km}$ is discussed for two different meteoroid sizes, and while being modest in scope, nevertheless provides a detailed overview of the main aspects of the flow regimes.

\section{FUNDAMENTALS AND BACKGROUND}

\subsection{Physical processes - formation of the hydrodynamic shielding, initial radius and shocks}

Following the initial sputtering regime (Rogers, Hill \& Hawkes 2005), it is possible to recognize three distinct stages of the early evolution of the sporadic overdense meteor train at lower altitudes (below $\sim 100 \mathrm{~km}$ ), beginning with the initial ablation and shock wave formation, and ending with the ambipolar diffusion and chemical removal of electrons from a thermalizing trail. Fig. 1 covers some of the features of the items under discussion, and we will refer to it repeatedly during our discussions.

Meteor trails are classified as underdense, transitionally dense or overdense, depending on their so-called line density $(q)$, or number of electrons per unit length of the trail. Electron densities in the plane perpendicular to the trail are integrated into the line-density calculation. By standard definition, transitional meteors have line densities in the range $2.4 \times 10^{14}$ to $10^{16}$ electrons $\mathrm{m}^{-1}$, while underdense and overdense meteors are those on the lower and upper ends, respectively, of the transitionally dense trail densities (McKinley 1961; Poulter \& Baggaley 1977, 1978). Here, we further describe the overdense meteors as particles with diameter between approximately $4 \times 10^{-3} \mathrm{~m}$ and up to small-sized fireballs (the latter size 
corresponding to or exceeding the electron line density of $q \sim 10^{19}$ electrons $\mathrm{m}^{-1}$; e.g. Sugar 1964).

We will now sequentially discuss the various stages of initial trail formation. In the first stage, meteoroids ablate due to high-energy hypervelocity collisions with surrounding atmospheric molecules (Öpik 1958; McKinley 1961; McNeil, Lai \& Murad 1998; William \& Murad 2002; Vondrak et al. 2008). The high-temperature ablated and ionized meteor atoms and electrons, together with ionized and dissociated atmospheric atoms, explosively form a dynamically stable meteor trail cylindrical volume with an initial radius $r_{0}$, which is approximated as quasi-neutral plasma that can subsequently be observed by meteor radars (McKinley 1961; Baggaley \& Fisher 1980; Jones 1995; Räbinä et al. 2016). Here, the term initial radius refers to the half-width of the initial (assumed) Gaussian distribution of the ions (or in the case of radio studies, electrons) that has 'instantaneously' and adiabatically formed immediately after the passage of the meteoroid, where the volume density of the free electrons is a function of meteoroid mass, size and ionization coefficient (e.g. Jones 1997; Jones \& Halliday 2001; Weryk \& Brown 2013). The adiabatic formation of the initial trail with radius $r_{0}$ is accompanied by turbulence generated in the meteor wake ( $T \leq 10000 \mathrm{~K}$ ), driven by the local flow field velocity, temperature and density gradients (Lees \& Hromas 1962). This process is completed within less than the first millisecond and it takes place after the formation and radial expansion of the cylindrical shock wave that will be discussed shortly. However, the density of ionized atoms and electrons in the meteor trail depends on the ionization coefficient (Kaiser 1953; Weryk \& Brown 2012, 2013). The majority of meteor radars, especially the lower power ones, detect electrons from the specularly reflecting meteor train (McKinley 1961; Hocking et al. 2016), while a smaller number of higher power radars can obtain reflections directly from the head and in non-specular mode. For the specularly reflecting scenario, overdense meteor trails in particular can be generally treated as metallic cylinders (Poulter \& Baggaley 1977, 1978) due to the high electron density and negative dielectric constant associated with plasma.

Ablated meteoric atoms have velocity-dependent kinetic energies that may reach several hundred electron volts (eV) (Baggaley 1980). The energy of collisionally released free electrons approaches several eV (e.g. see Baggaley 1980; Hocking et al. 2016 for discussion). The ion energy is converted to intensive heating of the flow field around the meteoroid and also of the ambient atmosphere. Note that our terminology with respect to the meteor-generated shocks attempts to reconcile hypersonic and the early meteor shock wave nomenclature (e.g. Bronshten 1965) in light of differences between meteors and a much slower hypersonic flow associated with typical re-entry vehicles.

It is important to emphasize that prior to the first stage of the meteor train evolution (Fig. 1), the onset of hydrodynamic shielding (Popova et al. 2001) at higher altitudes (as a precursor to the appearance of the ablation amplified meteor shock front) greatly affects the consideration of the hypervelocity flow in the front of and around the meteoroid (e.g. see Jenniskens et al. 2000; Gritsevich 2008). Moreover, the formation of the hydrodynamic shielding (sometimes referred to as the vapour cap), whose pressure and density are proportional to the cube of the meteoroid velocity (e.g. Öpik 1958; Bronshten 1983; Boyd 2000; Jenniskens et al. 2000; Popova et al. 2000; Campbell-Brown \& Koschny 2004), will alter the flow regime considerations (Boyd 2000; Popova et al. 2001) and Knudsen number (Josyula \& Burt 2011), shifting the freemolecular flow to higher altitudes, subsequently resulting in the formation of a meteor shock wave front $(T \gg 10000 \mathrm{~K})$ and a related cylindrical shock wave (Fig. 1) at higher altitudes (Jenniskens et al. 2000). This region consists of both reflected atmospheric constituents and collisionally ejected meteor atoms and ions. It also exhibits strong velocity-dependent density gradients near the meteoroid (Popova et al. 2000, 2001) and may be more than two orders of magnitude larger than the characteristic meteoroid dimensions.

Moreover, a single collision of an atmospheric molecule with the surface of a meteoroid may eject up to 500 meteoric atoms and molecules (Jenniskens et al. 2000); some which attain axial velocities 1.5 times higher than the parent meteoroid (Rajchl 1969). The hydrodynamic shielding becomes effective when the mean free path within the vapour cloud is approximately an order of magnitude smaller than the radius of the meteoroid (Popova et al. 2000). The meteor shock wave is formed when the hydrodynamic shielding is compressed (at lower altitudes), such that the changes in velocity, temperature and density are essentially a discontinuity.

The observational evidence indeed shows that the meteor bow shock (initial shock envelope associated with hypersonic flows) and the cylindrical shock wave (essentially approximated as a blast wave from the line source that depends on the amount of energy deposited per unit length (Lin 1954)) appear much earlier than predicted by classical gas dynamics theory (e.g. Rajchl 1969; Bronshten 1983; Brown et al. 2007; Silber \& Brown 2014). This occurs before the onset of the continuum flow (e.g. Probstein 1961; Bronshten 1983) and for most meteoroids takes place in the lower region of the transitional flow regime. This is especially relevant for overdense meteors discussed in our study. We expand this topic in more detail in the sections to follow.

The atmospheric gases swept behind either the hydrodynamic cap or, at lower altitudes, overdense meteor shock wave front (Fig. 1 and Fig. S1) are dissociated and ionized. The high-energy inelastic collisions of atoms or molecules behind the shock front and in the flow field result in a change of internal state and velocity of atomic and molecular species (Schunk \& Nagy 2009). Furthermore, these collisions usually involve the exchange of translational, rotational and vibrational energy, leading to the subsequent formation of new species (i.e. Brun 2009; Berezhnoy \& Borovička 2010). During elastic collisions in the overdense meteor wake, which occur in the 'lower' energy regime, the momentum and kinetic energy of the colliding particles are conserved and only translational energy exchange takes place. We are, however, not concerned with the processes that occur much farther back in the meteor wake and within the initially formed volume of the meteor train. This is because these processes do not contribute to appreciable removal of electrons from the overdense meteor train. Consequently, in this work, we are mainly interested in the processes that occur in the expanding high-temperature meteor train boundary with the ambient atmosphere. The processes within the wake and immediate train of the meteor trail have been discussed by Menees \& Park (1976), Park \& Menees (1978) and Berezhnoy \& Borovička (2010).

In the initial phase of the overdense meteor trail evolution, the ablated meteor plasma radiative energy loss takes place during the collisional deceleration, where the ablated plasma and the initially entrained and modified ambient gas stops within several hundred metres (Jenniskens et al. 2004). These processes coincide with what we define in this paper as the first stage of the overdense meteor trail evolution. This dynamic evolution of the high-temperature-ablated plasma and vapour exhibits rapid and highly turbulent initial flow in the meteor wake, which leads to the adiabatic formation of the more 
dynamically stable meteor trail volume with the initial radius $r_{0}$ (e.g. see Lees \& Hromas 1962; Jones 1995; Jones \& Campbell-Brown 2005; Hocking et al. 2016).

As shown in Fig. 1, the formation of the initial meteor trail is preceded by the cylindrical shock wave (the latter depends on the pressure ratio behind the shock and the ambient atmosphere, as a function of meteoroid size, velocity, ablation rate and Knudsen number; these are discussed in detail in Sections 3 and 4 and in Supporting Information). The cylindrical shock wave rapidly merges with the bow shock wave and expands radially with velocities significantly lower than the entry velocity of the meteor (in the considered meteor velocity range, e.g. see Tsikulin 1970). However, the cylindrical shock wave (discussed in detail further in the text) is sufficiently strong that it results in a near-instantaneous rise in temperature immediately behind the shock front that is of the order of several thousand Kelvin.

The second stage of high-temperature overdense meteor trail evolution is characterized by onset of ambipolar diffusion (Francey 1963; Pickering \& Windle 1970) that takes effect immediately after the explosive formation of the initial meteor trail volume (Fig. 1). It should be noted that the rate of ambipolar diffusion is a function of temperature and pressure (Hocking, Thayaparan \& Jones 1997).

The initial exchange and equilibration of translational, rotational and vibrational energy between atmospheric and ablating meteor constituents trapped within the flow-field brings the temperature in the wake of the meteor train (and initially formed meteor trail volume with radius $r_{0}$ ) down to about $4400 \mathrm{~K}$ (Jenniskens et al. 2004). Additionally, in the aforementioned important study, Jenniskens et al. (2004) found a marginal rise in temperature with decreasing altitude. More importantly though, they observed comparatively constant temperatures in the velocity range between 35 and $72 \mathrm{~km} \mathrm{~s}^{-1}$ and masses between $10^{-5}$ and $1 \mathrm{~g}$. The lower end of the spectrum of mass values reported by Jenniskens et al. (2004) is consistent with strong underdense meteors, while the upper end of the reported values corresponds to overdense meteor parameters.

Furthermore, the authors established that faster and more massive meteoroids produce larger emission volume but not a significantly higher air plasma temperature. Comparing their data with fireball temperatures obtained earlier, Jenniskens et al. (2004) concluded that the variation of meteor plasma emission temperatures for meteoroids in the range of masses between $10^{-5}$ and $10^{6} \mathrm{~g}$ is only up to several hundred Kelvin. Indeed, while surprising, such behaviour can be easily understood in terms of energy loss to molecular ionization, dissociation and hyperthermal chemical reactions in hypersonic reactive flows (Zel'dovich \& Raizer 2002; Anderson 2006; Brun 2009).

Moreover, observations show that it takes a few seconds for the temperature in a fireball wake with a visual magnitude of -12 to cool down from $4500 \mathrm{~K}$ down to $1200 \mathrm{~K}$, while for a typical overdense meteor with $M_{\mathrm{v}}=-3$, it takes $\sim 0.1 \mathrm{~s}$ (Jenniskens 2004). This reported meteor train cooling time is a significant development in understanding of the early meteor trail evolution, along with the observed and reported temperature values, because it allows a substantial amount of time for large-scale hyperthermal chemistry to take place on the boundary of the expanding overdense meteor train. More energetic and perhaps more complex sets of hyperthermal chemical reactions that occur inside the meteor train were discussed by Menees \& Park (1976), Park \& Menees (1978) and Berezhnoy and Borovička (2010). The implications of this will be discussed further shortly.

\subsection{Hyperthermal chemistry within the trail}

We now turn to issues of chemistry. While the chemistry within the trail is fairly well understood for the case that the trail has cooled down to ambient atmospheric temperatures (referred to as 'thermalized chemistry', e.g. Baggaley 1978, 1979; Plane 2012, 2015), there may also be substantial chemistry in the early stages of the trail formation, when temperatures are still very hot. This has not been explored as thoroughly as the thermalized chemistry, but has the potential to have significant impact on the life-cycle of the trail. This chemistry can occur in various places, including in association with the shocks (e.g. Zel'dovich \& Raiser 2002), within the trail, and (notably) on the edge of the trail. These processes can potentially result in rapid electron removal from the boundaries of the post-adiabatically expanding high-temperature meteor train. Eventually, this short period of initial electron removal terminates relatively rapidly, and then ambipolar diffusion takes over until the time at which thermalized chemistry starts to play the dominant role of electron removal (Baggaley \& Cummack 1974; Baggaley 1978). However, the time taken for the hot parts of the trail to settle down to ambient temperature is still the subject of considerable uncertainty, and can have profound effects on diffusion rates. Hocking et al. (2016, appendix C) suggest that this may require that the net diffusion rates of the trail should be the geometric average of the diffusion coefficients of the hot plasma and the ambient background atmosphere. The validity of this assumption is critically dependent on the rate at which temperature equilibrium in the trail is achieved. While once considered near-instantaneous, this is now questionable. We will return to this point later: for now, we concentrate on the chemical processes that occur while the region is still hot, which we take to be prior the first $0.1-0.3 \mathrm{~s}$, which is required for the overdense trail to thermalize.

We now look at the implications for chemistry within the hightemperature regime. This high-temperature meteor train expands post-adiabatically into the ambient atmosphere, modified by the cylindrical shock wave.

These processes then enable the temperature driven oxidation of meteoric metal ions in the trail boundary by the ambient oxygen that survives the passage of cylindrical shock wave some distance away from the high-temperature meteor train and also the shock dissociated product $\left(\mathrm{O}_{2}\right)$ of ozone $\left(\mathrm{O}_{3}\right)$ in the meteor near-field. The reaction is expressed as $\mathrm{M}^{+}+\mathrm{O}_{2} \rightarrow \mathrm{MO}^{+}+\mathrm{O}$, where $\mathrm{M}^{+}$ is a common meteoric metal ion. The process is generally completed in $10^{-3}-10^{-1} \mathrm{~s}$, for altitudes between 80 and $100 \mathrm{~km}$. The observational evidence of much slower thermalization of the meteor trains (Jenniskens et al. 2004) corroborates the presence of a high-temperature environment conducive to hyperthermal chemistry. Notably, the production of metal oxide ions will be governed by the second Damköhler number (which represents the ratio of the chemical reaction rate to the ambipolar diffusion mass transfer rate) and the temperature $(1500 \mathrm{~K}<T<3000 \mathrm{~K})$, with the highest yield at about $2500 \mathrm{~K}$ (Berezhnoy \& Borovička 2010). We will discuss this in detail in the next section.

The third and final stage of the trail development takes place within the boundary of the almost thermalized ambipolarly diffusing meteor train sketched in Fig. 1. Here, hyperthermally produced meteor metal oxide ions rapidly remove electrons in the almost thermalized train, through temperature-independent dissociative recombination, $\mathrm{MO}^{+}+\mathrm{e} \rightarrow \mathrm{M}+\mathrm{O}$ (Plane 2012, 2015). The reaction terminates when $\mathrm{MO}^{+}$is consumed. Depending on the available raw material $\left(\mathrm{MO}^{+}\right)$, this reaction may have a noticeable impact on the lifetime of the trail by removing electrons in this early phase. 
In the following sections, we examine in more detail the evidence for hyperthermal chemistry that has so far been only briefly summarized. We also refer to the modelling work used to illustrate the effect of shock waves; details are included in Section 4.3 and Supporting Information, where we present an even more comprehensive discussion.

\section{LINKING THE SHOCK WAVES AND METEOR TRAIN - ATMOSPHERE HYPERTHERMAL CHEMISTRY}

The meteor cylindrical shock waves have the strongest effect in the region of the ambient atmosphere relatively close to the adiabatically formed meteor trail volume. In this region, defined as the characteristic or blast radius $R_{0}$ (ReVelle 1976; Silber, Brown \& Krzeminski 2015), the initial energy deposition per unit length is the largest, because overdense meteor cylindrical shock waves are in principle approximated as explosive line sources (e.g. see Lin 1954; Bennett 1958; Jones et al. 1968; Tsikulin 1970). That is primarily due to the fact that there is an almost instantaneous release of a comparatively large quantity of energy in a limited geometrically defined space (Steiner \& Gretler 1994). We note that $R_{0}$ (the characteristic or blast radius) is different to the previously defined $r_{0}$ (meteor trail volume radius). The relationship between the maximum energy deposition and the characteristic radius $R_{0}$ shown in Fig. 2 (ReVelle 1974, 1976) is expressed as

$R_{0}=\left(E_{0} / p_{0}\right)^{0.5}$.

Here, $E_{0}$ is the energy deposited per unit path length (which in the case of a meteoroid is the same as the total aerodynamic drag per unit length) and $p_{0}$ is the ambient pressure (e.g. Silber et al. 2015). The term characteristic radius is used only in reference to strong shock waves, when the energy release $\left(E_{0}\right)$ is sufficiently large that the internal energy of the ambient atmosphere is negligible (Lin 1954; Hutchens 1995). Fig. 2 shows the initial radius $\left(r_{0}\right)$ of bright overdense meteors (Baggaley \& Fisher 1980; Ceplecha et al. 1998) and the radius of the overdense meteor trail after $0.3 \mathrm{~s}$. Those are compared with the characteristic radius $\left(R_{0}\right)$ associated with the constant energy deposition of 100 and $1000 \mathrm{~J} \mathrm{~m}^{-1}$, for the altitude $80-100 \mathrm{~km}$. The aforementioned energies represent the velocity, size and composition dependent peak energy depositions (e.g. see Zinn, O'Dean \& ReVelle 2004; Silber et al. 2015) for different sizes of overdense meteors ablating in that narrow region of MLT. It is readily seen that $R_{0}$ is always greater than $r_{0}$ for constant energy deposition values corresponding to overdense meteors in MLT, and it approximately matches or is greater than the radius of the ambipolarly expanded meteor trail volume after $0.3 \mathrm{~s}$ (depending on the choice of initial $r_{0}$ and the diffusion coefficient $D$ ). We will come back to this point later (Section 4.1).

However, the maximum effect on the ambient atmosphere, such as dissociation, is most dominant within the characteristic radius of $R_{0}$. After that, the shock wave attenuates rapidly and transitions to the acoustic regime within $10 R_{0}$ (ReVelle 1976; Silber 2014, 2015). The initial temperature behind an overdense meteor cylindrical shock wave is typically in the vicinity of $6000 \mathrm{~K}$ (as will be demonstrated in the following sections), sufficient to dissociate $\mathrm{O}_{2}$ and $\mathrm{O}_{3}$ within $R_{0}$.

Oxygen, regardless of its initial source (Dressler 2001), is the most likely molecule to react hyperthermally and rapidly with the ablated meteor ion in the boundary of the high-temperature meteor train (e.g. see Murad 1978). For the simplicity of the exposition, this paper consequently only focuses on the initial meteor train near-field $\left(\sim R_{0}\right)$ where the product of high-temperature oxidation
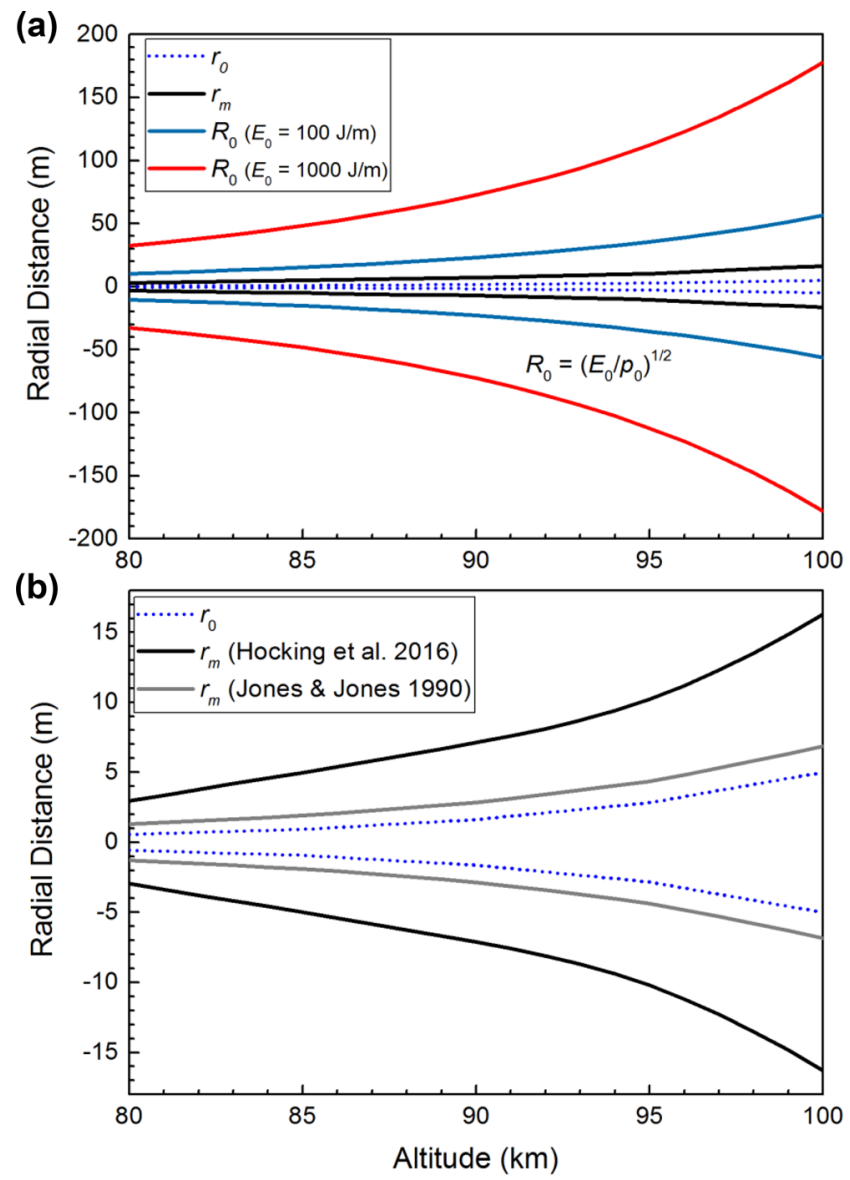

Figure 2. (a) Plotted are the initial radius $\left(r_{0}\right)$ of a typical bright overdense meteor (from Baggaley \& Fisher 1980) and the radius of the meteor $\left(r_{\mathrm{m}}\right)$ trail after $t=0.3 \mathrm{~s}$. These are compared to $R_{0}$ as a function of constant energy deposition (see equation 1) of 100 and $1000 \mathrm{~J} \mathrm{~m}^{-1}$ for altitudes from 80 to $100 \mathrm{~km}$. For $r_{\mathrm{m}}$ at $0.3 \mathrm{~s}$, we applied the geometrically averaged hot plasma and ambient atmosphere ambipolar diffusion coefficients as per Hocking et al. (2016, appendix C). (b) The initial radius $r_{0}$, plotted along $r_{\mathrm{m}}$ at $t=$ $0.3 \mathrm{~s}$. Shown here is the comparison between $r_{\mathrm{m}}$ as calculated in panel (a), and $r_{\mathrm{m}}$ as calculated using Massey's formula for the theoretical diffusion coefficient (Jones \& Jones 1990).

of meteor metal ions, along with subsequent dissociative recombination, is the only reasonably fast mechanism capable of removing electrons from the boundary of meteor trail in the initial stage of post-adiabatic ambipolar diffusion (Dressler 2001). Another important aspect examined in this work is the source of $\mathrm{O}_{2}$ (ambient or products of ozone shock dissociation) that dominates in the hightemperature rapid production of the meteor metal ions oxides that are subsequently responsible for the post-hyperthermal chemical removal of electrons from the boundary of the overdense meteor train.

To further examine these issues, we need to consider the pressure and temperature gradients in the flow field in and around the meteoroid and in the meteor wake relative to the ambient atmosphere, along with the dissociative behaviour, excitation and ionization potentials of atmospheric molecules. Furthermore, some aspects of high-temperature gas dynamics and chemistry involving both major and minor MLT species need to be further illuminated in order to understand the complex processes that take place on short time-scales in the boundary of the post-adiabatically expanding hot meteor train. 


\subsection{Initial shock and hyperthermal chemistry within high-temperature meteor trail}

Within the volume of vapour and plasma, beginning from the region behind the meteor shock front and enclosed by the envelope of the initial shock (see Fig. S1), various complex physicochemical processes, such as the high-temperature reactive and non-equilibrium flows, ionization, dissociation and excitation, take place at very small time-scales (Kogan 1969; Menees \& Park 1976; Park \& Menes 1978; Shen 2006; Brun 2009, 2012). The relative importance and the rates of those processes depend upon the temperature and density and the time-scales at which the relaxation between translational, rotational and vibrational energies take place.

The early chemical reactions occur as a result of almost instantaneous gas heating, which is caused by collisions with ablated and evaporated meteoric material. These collisions, however, are caused by initial 'instantaneous' compression and kinetic and radiative energy exchange behind the shock front (Anderson 2006). High-energy molecular and atomic collisions also occur (at temperatures generally far above the characteristic vibrational temperature of the diatomic molecule), followed by the equilibration between translational and internal degrees of freedom. This is superseded by subsequent dissociation, ionization and radiation in addition to various non-equilibrium chemical reactions (Panesi et al. 2011; Brun 2012).

Behind the initial meteor shock front (Fig. S1), velocitydependent ionization occurs rapidly, involving both impinging atmospheric constituents and ablated meteoric atoms. Notably, meteoric metal atoms (e.g. Fe and $\mathrm{Mg}$ ) will ionize more efficiently due to their lower ionization potential (Dressler 2001). Competing ionization processes take place, such as ionization by molecular and atomic collisions, electron impact and ion impact ionization, in addition to photoionization; the respective reactions and required energies are given and discussed by Lin \& Teare (1963), Park (1989) and Starik, Titova \& Arsentiev (2009). Moreover, behind the shock wave front, the vibrational temperature depends on vibrational relaxation rates, as well as coupling of the vibrational relaxation and dissociation of molecules (Zabelinskii et al. 2012). However, the rate of dissociation behind the shock wave is reduced when the vibrational temperature has not equilibrated with the translational temperature (Boyd, Candler \& Levin 1995).

The translational temperature, which increases rapidly behind the shock front (e.g. Boyd 2000; Sarma 2000; Zinn et al. 2004; Zinn \& Drummond 2005), decreases quickly as the rotational and vibrational energies are raised. The vibrational modes take longer to equilibrate with translational and rotational temperatures. In the case of the reactive flow around overdense meteors in the MLT region, the comparison of chemical and hydrodynamic time-scales during the initial stages of the flow within the shock layer, as depicted in Fig. S1, indicates that the equilibrium is still not reached in the initial flow field behind the shock front because the chemical reaction time-scales are longer than the hydrodynamic time-scale (Berezhnoy \& Borovička 2010). However, after equilibrium between the various energy modes is established, further energy is consumed by dissociation and ionization (Hurle 1967), followed by the beginning of various thermally driven chemical reactions with different characteristic times (Sarma 2000; Brun 2012).

Further down the meteor axis, within the high-temperature region in the meteor wake, shock-modified reactive flow of ablated vapour and plasma occurs, carrying the entrained excited, dissociated and ionized atmospheric constituents. This is an ideal environment for the formation of nitric oxides, as was discussed by Menees \& Park (1976) and Park \& Menees (1978).

However, it is useful to recall at this moment that the dissociation and ionization threshold energies of $\mathrm{N}_{2}$ and $\mathrm{O}_{2}$ are very high (Massey \& Bates 1982; Rees 1989). Comparatively, meteoric metal atoms have low ionization potentials and can be ionized efficiently, as mentioned earlier (relative to atmospheric molecules and atoms), in high-velocity neutral collisions (Dressler 2001).

It must be emphasized at this point that in general, no appreciable electron removing reactions between the meteoric constituents take place within the expanding meteor train (Berezhnoy \& Borovička 2010). This is important as it indicates that the processes responsible for the initial rapid and short-lasting electron removal occur mainly on the boundary of the meteor train.

Within the high-temperature meteor trail, nitric oxide is generally formed by a hyperthermal reaction between available $\mathrm{N}_{2}$ and $\mathrm{O}$ within the meteor trail volume with the initial radius $r_{0}$, where $\mathrm{N}_{2}+\mathrm{O} \rightarrow \mathrm{NO}+\mathrm{N}$. The reaction proceeds when the temperature is in the range between 2000 and $10000 \mathrm{~K}$ (Menees \& Park 1976). Below $2000 \mathrm{~K}, \mathrm{NO}$ is further produced by the reaction $\mathrm{N}+\mathrm{O}_{2} \rightarrow$ $\mathrm{NO}+\mathrm{O}$. The first reaction is endothermic, while the second reaction is temperature independent, and will proceed inside the volume of the adiabatically formed meteor train with the initial radius $r_{0}$ until almost all supplies of $\mathrm{N}$ atoms are exhausted (including small quantities of $\mathrm{O}_{2}$ within the high-temperature meteor train). It should be noted that the reverse of the first reaction occurs at lower temperatures, which removes N and NO from the flow (Menees \& Park 1976).

From the perspective of the high-temperature meteor train chemistry, this is very relevant because there will be a negligible amount of remaining $\mathrm{N}$ within the meteor train volume to engage in reactions outside the meteor train boundary.

A more detailed study and description of the thermally driven chemical reactions in high-temperature meteor train is given by Berezhnoy \& Borovička (2010). A detailed analysis of the reactions of atomic and molecular metastable species behind the shock wave is presented by Starik et al. (2009). The authors presented an extensive list of reactions and reaction rates for the range of excited dissociated and ionized atmospheric constituents, which serves to further illuminate the very complex and previously difficult-to-model chemical dynamics of the shock wave environment. We can now go back and examine the dynamics and physicochemical effects of the meteor cylindrical shock waves.

\section{EVALUATION OF SHOCK WAVE EFFECTS}

\subsection{Dynamic and physicochemical effects of overdense meteor cylindrical shock waves}

Depending on Knudsen number, velocity, size and composition of overdense meteors, the energy deposited per unit path length may reach as high as several thousand $\mathrm{J} \mathrm{m}^{-1}$ (Zinn et al. 2004; Silber et al. 2015). This energy, assumed to be released instantaneously along the axis of meteor propagation, drives the radial expansion of the cylindrical shock (Zel'dovich \& Raizer 2002). In treatment of the cylindrical shock waves, it is assumed that all of that energy is deposited almost instantaneously in the cylindrical volume of the atmosphere with radius $R_{0}$ (Lin 1954; Plooster 1968; Tsikulin 1970), as mentioned earlier.

It is well established that the speed of the shock wave depends only on the difference in pressure of the region where the energy is deposited relative to the pressure in the ambient gas (e.g. Hurle 
1967). Thus, if the velocity (and consequently the strength) of the meteor bow and vapour cylindrical shock waves are to be determined, the pressure behind the shock front or vapour pressures in the compressed flow field region behind the meteoroid are important parameters and must be known (Bronshten 1983; Zel'dovich \& Raizer 2002; Anderson 2006). While we can distinguish, for pedantic purposes, between the two main types of the cylindrical shock waves (the initial bow shock and the ablation amplified recompression cylindrical shock wave; see Supporting Information) during the initial shock evolution (e.g. Hayes \& Probstein 1959; Bronshten 1983; Sarma 2000), that distinction cannot be made outside of the immediate region of maximum energy deposition with the characteristic radius $R_{0}$, as these two types of shock waves will coalesce rapidly. The pressures in the stagnation region behind the region of the blunt shock front ahead of and on the axis of the meteor can be determined based on the meteoroid characteristics (Bronshten 1965; Tsikulin 1970).

Let us consider and compare the bow (initial or primary cylindrical shock wave) and the ablation amplified recompression cylindrical shock wave (Sarma 2000) which, in the case of ablating meteoroids, is defined as the ablational or vapour cylindrical shock wave (Bronshten 1983). In simple terms, the bow shock wave strength and the velocity of radial expansion will depend on the meteoroid velocity, the initial flow translational temperatures, and the subsequent pressures behind the shock front in the front of the meteor. It will also strongly depend on the specific heat ratios, as they will dictate the geometry of the blunt region (Anderson 2006). The recompression shock wave, while common in all hypersonic bodies (e.g. Hayes \& Probstein 1959; Sarma 2000), will be different for overdense meteors in the transitional and continuum flow regimes, because it will depend directly on the amount of ablated material from the meteoroid (Bronshten 1983; Zinn et al. 2004). As importantly, the strength of the cylindrical shock originates from the compressed flow field around and behind the meteoroid (e.g. the neck region of the flow field) and depends on both the flow temperature and the vapour and plasma pressure at the neck (the region of the maximum gas and plasma compression behind the meteoroid; Fig. S1).

For illustrative purposes, consider the dissociated ambient atmosphere, initially swept behind the meteor shock front. It is compressed along with ablated meteoric plasma and vapour (Popova et al. 2001) and still has temperature significantly greater than $10000 \mathrm{~K}$ in the immediate flow field behind the meteor (Boyd 2000; Jenniskens et al. 2000; Fig. S1). In that region, the flow field converges and is compressed to pressures several orders of magnitudes higher than the ambient atmospheric gas. Moreover, the pressure increase relative to the ambient gas is amplified by ablation, which frees significantly more than $10^{16}$ ions and atoms per meter, for the case of an average overdense meteor. Considering that the ambient gas temperature is about $200 \mathrm{~K}$, and the temperature in the flow field behind the meteoroid exceeds $10^{4} \mathrm{~K}$, it can be shown using the equation of state for a gas that even without ablation, or a volume reduction, the pressure increase in the flow field behind the meteoroid exceeds 50 times that of the ambient gas.

This problem was first considered by Dobrovol'skii (1952), and while initially dismissed by relevant investigators at the time, it has been proven valid (Bronshten 1983). Let us consider the loss of meteoroid kinetic energy, which can be written using the following expression:

$\frac{\mathrm{d} E}{\mathrm{~d} t}=\frac{d}{\mathrm{~d} t}\left(\frac{m v^{2}}{2}\right)=m v \frac{\mathrm{d} v}{\mathrm{~d} t}+\frac{v^{2}}{2} \frac{\mathrm{d} m}{\mathrm{~d} t}$.
Here, the first term on the left represents the energy lost per unit of time and $m$ and $v$ are the meteoroid mass and velocity, respectively (e.g. Romig 1964; Gritsevich \& Koschny 2011). Dividing both sides of equation (2) by the velocity ( $v$ ) (Bronshten 1983) the energy deposition per unit path length can be obtained:

$\frac{\mathrm{d} E}{\mathrm{~d} l}=m \frac{\mathrm{d} v}{\mathrm{~d} t}+\frac{v}{2} \frac{\mathrm{d} m}{\mathrm{~d} t}$.

As indicated by Bronshten (1983), the first term on the right in equation (3) is the energy used to form the bow shock wave, assuming no ablation. The second term then is the energy partitioned to the ablation and lost to the ablated vapour per unit length. It can be shown that the second term is utilized to describe the formation of the comparatively stronger cylindrical vapour shock wave. To demonstrate this, we need to consider the ratio of the differentiated terms on the RHS in equation (3). The ratio of the two terms on the right in equation (3), $\frac{\frac{v}{2} \frac{\mathrm{d} m}{\mathrm{~d} t}}{m \frac{\mathrm{d} v}{\mathrm{~d} t}}$, was compared by Dobrovol'skii (1952), with certain simplifying assumptions (see Bronshten 1983 for a discussion), and the results indicated that the second term is significantly bigger. Depending on the meteoroid velocity and the rate of ablation, the second term may be more than two orders of magnitude larger than the first term, especially for the higher velocities.

As discussed earlier, this meteoroid-deposited energy can be equated with the blast wave from exploding cylindrical line sources as discussed by Lin (1954), Bennett (1958), Jones, Goyer \& Plooster (1968) and Plooster (1970). Lin (1954) presented the solution for the cylindrical shock wave produced by instantaneous energy release, where he defined the radius of the cylindrical shock wave and determined its rapid decay as a function of time. The shock envelope behind the meteoroid is a function of the aerodynamic drag, initial density and meteoroid velocity. Bronshten (1983) offers a detailed meteorcentric discussion of the problem.

The pressure ratios of the ablated, vapourized meteoroid and plasma in the flow field $(p)$ to that of the ambient atmosphere $\left(p_{0}\right)$ for an average size of overdense meteors with $1 \mathrm{~cm}$ radius, as evaluated by Bronshten (1983) lie in the range $10^{2}<p / p_{0}<10^{4}$. This is particularly true for events with velocities exceeding $30 \mathrm{~km} \mathrm{~s}^{-1}$, where much more energy is transferred to the flow field vapour and plasma behind the shock front, than is spent on the ablation process. It is the dispersion of this ablated and pressurized 'vapour' in the front of the meteoroid that amplifies the shock wave (Dobrovol'skii 1952; Bronshten 1983; Zinn et al. 2004).

Consequently, it can be seen that the ablation and vapour amplified cylindrical shock wave is, in the case of intensely ablating, fast meteoroids, significantly stronger than the initial bow shock wave in the absence of ablation. In principle, however, the two cylindrical shock waves (initial bow, and ablation amplified recompression or cylindrical shock wave) rapidly merge and cannot be distinguished, as mentioned earlier.

We can use this value of $p / p_{0}$ to estimate the strength of the typical overdense meteor cylindrical shock wave, assuming that we know the pressures and temperatures of the vapourized and ablated material, as well as the flow field surrounding the entrapped atmospheric dissociated molecules, in the neck region behind the meteoroid (Fig. S1).

For the purpose of simplification, we assume that the pressures of the ablated high-temperature vapour and plasma around and behind the meteoroid exceed the ambient pressure by at least two orders of magnitude (Bronshten 1983). In principle, this might be a significantly understated value as demonstrated in early studies 
(Bronshten 1965, 1983) and it can be reasonably interpreted to correspond more to the pressure ratios associated with transitional meteors (e.g. Popova et al. 2000, 2001). However, we use it here for expository discussion of the nature of the problem. Furthermore, it is reasonable to assume that the pressure in the neck region of the flow field, behind the meteoroid, due to the ablation, dissociation and high-temperature flows will be similar to pressures in the region around the stagnation point (Fig. S1), behind the initial shock front. Therefore, this gives us a reasonable tool to approximately evaluate the cylindrical shock velocity and strength that originates from the high-temperature compressed flows in the neck region (Fig. S1).

Thus, in order to evaluate the initial velocity and strength, and consequently the effects of the cylindrical shock waves, we need to know with reasonable accuracy the vapour and plasma pressure behind the cylindrical shock front in the flow field region behind the meteoroid where the shock wave is generated.

For the purpose of this exposition, we consider Brohnsten's (1983) discussion as a guide to approximate the pressure ratios of ablated vapour and the ambient atmosphere as $p / p_{0} \sim 100$ as discussed above. This is taken as the lowest value for overdense meteors with a size range discussed earlier for the purpose of evaluating the strength of the cylindrical shock wave (see chapter 3 , section 17 in Bronshten 1983 for discussion). The cylindrical shock wave velocity (or Mach number) can be easily obtained from the expression for the pressure behind the shock front that is generally evaluated using the Hugoniot relationship. It relates the vapour pressure behind the shock $(p)$ and the ambient pressure $\left(p_{0}\right)$, shock Mach number $\left(M_{\mathrm{sw}}\right)$ and the ratio of specific heats $(\gamma)($ e.g. Lin 1954; Jones et al. 1968; Tsikulin 1970):

$\frac{p}{p_{0}}=\frac{2 \gamma}{\gamma+1} M_{\mathrm{sw}}^{2}$

This relationship can be used in the region of the strong shock wave where $p>>p_{0}$ (Lin 1954; Jones et al. 1968). Another way to roughly estimate the shock wave velocity is by utilizing the vapour temperature and corresponding high thermal velocities, as demonstrated by Zinn et al. (2004).

Experimental observations agree with expression (4) in the region of the strong shock wave $\left(R_{0}\right)$ (e.g. Jones et al. 1968, Plooster 1970 and references therein) where empirically derived relations for the density, pressure and temperature ratios (see Zel'dovich \& Raizer 2002) are written as

$$
\begin{aligned}
\frac{\rho}{\rho_{0}} & =\frac{6}{1+5 M_{\mathrm{sw}}{ }^{-2}} \\
\frac{p}{p_{0}} & =\frac{7}{6} M_{\mathrm{sw}}^{2}-\frac{1}{6} \\
\frac{T}{T_{0}} & =\frac{1}{36}\left(7-{M_{\mathrm{sw}}}^{-2}\right)\left(M_{\mathrm{sw}}^{2}+5\right) .
\end{aligned}
$$

Here, $\rho_{0}$ and $T_{0}$ are the mean density and temperature ahead of the shock wave, respectively. The same parameters without subscript are the values just behind the shock front. Combining equations (5-7) (for details see Hurle 1967; Zel'dovich \& Raizer 2002), the temperature behind the shock wave can be calculated if the cylindrical shock wave Mach number or the pressure ratio are known. The discussion and theoretical treatment of shock wave Mach number along with the flow regimes are given in Section S1 (Supporting Information) in this paper.

Then, using the Rankine-Hugoniot relations $\left(p / p_{0} \approx 100\right)$, and assuming an ideal diatomic gas, the velocity of the cylindrical shock wave is calculated to be around Mach 9.3 and the temperature behind the shock is in the range of $6000 \mathrm{~K}$, assuming an ideal gas (see Hurle 1967 , where the value of $6020 \mathrm{~K}$ is suggested).

However, the actual value of temperature is lower, as the non-ideal gas behaviour affects the temperature values through the mechanisms (Anderson 2006) discussed earlier in the text and in considerable detail in Supporting Information. Moreover, in the MLT, the ratio of specific heats $(\gamma)$ will be also different, leading to the lower values of the calculated temperature (e.g. Viviani \& Pezzella 2015). Generally, below $95 \mathrm{~km}$, the pressures of the vapour and the rate of ablation for average overdense meteors will be in the above mentioned range, depending on velocity (Bronshten 1983; Boyd 2000). However, considering the much higher vapour pressure ratios for typical chondritic meteoroids behind the shock front, the velocity of the ablationally amplified cylindrical shock wave (within the $R_{0}$ region) may be significantly higher than our estimate. In reality, the Mach number of the cylindrical shock waves may easily reach or exceed Mach 20 for the upper sizes of overdense meteors with higher velocity and large energy deposition $\left(E_{0}>1000 \mathrm{~J} \mathrm{~m}^{-1}\right)$.

These high velocities bring the temperatures behind the cylindrical shock wave to the range of $6000 \mathrm{~K}$, even after taking into consideration a non-ideal gas specific heat ratio, and effects of relaxation and dissociation (Anderson 2006).

Importantly, such temperatures behind the cylindrical shock wave are sufficient for strong dissociation and excitation of atmospheric species within $R_{0}$, but will not be high enough for any appreciable ionization. Generally, at temperatures in the range of 3000-7000 K behind the shock front in a typical atmospheric diatomic gas, there is still no appreciable ionization. Under such conditions, the molecular vibrations are excited relatively quickly, and the thickness of the wave front is connected with the slowest relaxation process, namely molecular dissociation (Zel'dovich \& Raizer 2002).

Knowledge about these temperatures is the key to understanding the dominant chemistry in this region. Let us consider molecular oxygen first, which is inert below $800 \mathrm{~K}$ (Zel'dovich \& Raizer 2002). The dissociation energy for $\mathrm{O}_{2}$ is $5.12 \mathrm{eV}$ or about $59000 \mathrm{~K}$ (Bauer 1990). The rate of dissociation of oxygen behind the shock wave is a function of temperature, as shown by Ibraguimova et al. (2012). The heated $\mathrm{O}_{2}$ molecules begin to dissociate between approximately $2000 \mathrm{~K}$ and $4000 \mathrm{~K}$ at normal pressure, while above $4000 \mathrm{~K}$ almost all oxygen is dissociated (Bauer 1990; Anderson 2006). However, the dissociation temperature range is affected by the pressure, and thus in the MLT region, the dissociation takes place at lower temperatures (Bauer 1990). The dissociation of molecular oxygen in the atmosphere has been presented in detail by Nicolet \& Mange (1954) and will take place behind the strong shock wave, when the vibrational temperature is equilibrated with translational and rotational temperatures. As a comparison, the dissociation energy of $\mathrm{N}_{2}$ is $9.76 \mathrm{eV}$ (Bauer 1990). $\mathrm{N}_{2}$ dissociation starts slowly above $4000 \mathrm{~K}$ and is almost complete just above $9000 \mathrm{~K}$ (Anderson 2006; Fridman 2008).

Moreover, the thermal non-equilibrium chemical kinetics and dissociation of $\mathrm{O}_{2}$ behind the shock front has been investigated by Gidaspov, Losev \& Severina (2010). For typical cylindrical shock wave strengths discussed here, the time-scales for the dissociation of $\mathrm{O}_{2}$ will be closely in line with the typical time of vibrational excitation of two-atom molecules, and can be approximated by the Landau-Teller formula (Gidaspov et al. 2010 and references therein). Both vibration relaxation and dissociation time-scales decrease with increasing temperature, and their ratio for oxygen approaches unity in the region between 2000 and $4000 \mathrm{~K}$. For the purpose of this analysis, and based on the data and calculations 
presented in Nelson (1964), Bauer (1990) and Takayama (2012), and the rates given by Gidaspov et al. (2010) and Ibraguimova et al. (2012), it is reasonable to estimate that a significant proportion of $\mathrm{O}_{2}$ is dissociated within approximately $10^{-4} \mathrm{~s}$ behind the cylindrical shock wave passage within the $R_{0}$ blast region surrounding the initially formed meteor trail volume in the MLT region.

The implication of the above discussion is that behind the overdense meteor cylindrical shock wave in the near field region surrounding the meteor train boundary, and at related temperatures, most $\mathrm{O}_{2}$ will be dissociated in the proximity of the boundary of the initial meteor high-temperature train volume and less so towards the $R_{0}$, with only a negligible fraction of $\mathrm{N}_{2}$ dissociated. However, it must be emphasized again, that while we are discussing the generalized case, the actual amount of surviving $\mathrm{O}_{2}$ depends primarily on the energy deposition that may vary across the overdense meteor size spectrum.

For comparative purposes and in order to investigate the flow and temperature fields around a meteor, we have numerically modelled the hypersonic flight dynamics for two non-ablating spherical bodies $\left(\right.$ diameter $=2.5 \times 10^{-2}$ and $\left.1 \times 10^{-1} \mathrm{~m}\right)$ with velocity $35 \mathrm{~km} \mathrm{~s}^{-1}$ and at $80 \mathrm{~km}$ altitude (Section 4.3).

\subsection{Ozone dissociation behind the cylindrical shock wave}

Ozone in its native form cannot survive the effects of the meteorgenerated cylindrical shock waves because of its characteristic temperature sensitivity (Schumacher 1960; Jones \& Davidson 1962; Benson \& Axworthy 1965; Michael 1971) and its low dissociation potential (Center \& Kung 1975; Endo et al. 1979), which is much lower than that for $\mathrm{O}_{2}$. However, as a result of such properties of ozone, a variety of energetic species can be produced through its dissociation, with modest amounts of input energy (Zel'dovich \& Raizer 2002). Let us consider what happens with ozone at those initial kinetic temperatures in the range of $6000 \mathrm{~K}$ behind the cylindrical shock front, in the meteor trail near-field. The process of collisional dissociation of ozone with sufficiently energetic particles in the high-temperature region behind the shock wave usually corresponds to the reaction:

$\mathrm{O}_{3}+\mathrm{M} \Leftrightarrow \mathrm{O}_{2}+\mathrm{O}+\mathrm{M}$,

where $\mathrm{M}$ is any atmospheric molecule or atom $\left(\mathrm{O}_{2}, \mathrm{~N}_{2}, \mathrm{O}, \mathrm{N}\right)$. Ozone dissociation (Jones \& Davidson 1962; Center \& Kung 1975; Fridman 2008; Konnov 2012) is highly temperature dependent, and at meteor shock wave temperatures in the MLT region, the dissociation times are in the order of $10^{-5} \mathrm{~s}$. The ozone dissociation time decreases with increasing temperature $T_{0}$. It should be also noted that while the dissociation of ozone is an endothermic reaction, the formation of ozone is exothermic. However, the higher temperatures in the vicinity of the boundary region of the meteor train impede new ozone production in the shock heated gas and meteor plasma (from the initially dissociated oxygen and ozone products). The kinetics of the excited products of dissociation of $\mathrm{O}_{3}$ and $\mathrm{O}_{2}$ is discussed by Yankovsky \& Manuilova (2006). Comparing the dissociation potentials of oxygen and ozone, we can see that relative to oxygen dissociation energy of $5.12 \mathrm{eV}$, the dissociation energy of ozone is significantly less, at about $1.04 \mathrm{eV}$ (Bauer 1990).

Above $1500 \mathrm{~K}$, ozone dissociation times are in the order of $\mu \mathrm{s}$ (Jones \& Davidson 1962; Benson \& Axworthy 1965; Johnston 1968; Michael 1971; Center \& Kung 1975; Konnov 2012; Peukert et al. 2013). Endo et al. (1979) investigated the thermal dissociation of $\mathrm{O}_{3}$ behind a shock wave with temperatures between 600 and $1100 \mathrm{~K}$, where ozone dissociates into the low-energy triplet $\mathrm{O}_{2}\left(\mathrm{X}^{3} \Sigma_{g}^{-}\right)$and the low-energy molecular oxygen $\mathrm{O}\left({ }^{3} \mathrm{P}\right)$ with activation energy of $0.98 \mathrm{eV}$.

The decomposition of ozone in flames, recently modelled by Konnov (2012), is known to produce the triplet $\mathrm{O}_{2}\left(\mathrm{X}^{3} \Sigma_{\mathrm{g}}^{-}\right)$and $\mathrm{O}\left({ }^{3} \mathrm{P}\right)$, and yields small quantities of the singlet $\mathrm{O}_{2}\left(\mathrm{a}^{1} \Delta_{\mathrm{g}}\right)$. At shock wave temperatures and time-scales, the reaction: $\mathrm{O}_{2}\left(\mathrm{a}^{1} \Delta_{g}\right)+\mathrm{O}_{2} \rightarrow$ $\mathrm{O}_{3}+\mathrm{O}$, along with other ozone-forming reactions, will not proceed. Moreover, the collisional efficiency of $\mathrm{O}_{3}$ is assumed to be 2.5-3 times higher than that for $\mathrm{O}_{2}$ (which is in turn more collisionally efficient than $\mathrm{N}_{2}$ ). Notably, atomic oxygen has a collisional efficiency that is 4-5 times more than that of $\mathrm{O}_{2}$ (Makarov \& Shatalov 1994; Luther et al. 2005; Konnov 2012). However, considering the meteor cylindrical shock wave temperatures and the associated short time-scales, a significant amount of $\mathrm{O}_{2}$ that originates from the $\mathrm{O}_{3}$ shock dissociation will survive closer to the meteor trail boundaries (within $R_{0}$ ). This is due mostly to the finite energy budget and finite time-scales available for dissociation, which exist behind the typical overdense meteor cylindrical shock waves. Of course, the same consideration can be applied to fireball-type of events (as demonstrated by Zinn et al. 2004) or on the opposite end of the size-spectrum, to transitional meteors, where the strength of the cylindrical shock remains uncertain (in cases when such shocks exists). As will be demonstrated shortly, this is very important, as surviving oxygen is available for the high-temperature reactions with meteor metallic ions and is of critical importance for determining any potential role of these processes in early electron removal.

At overdense meteor cylindrical shock wave temperatures, however, dissociation of ozone will yield the presence of both excited and ground state particles of both $\mathrm{O}_{2}$ and $\mathrm{O}$, where the excited species $\mathrm{O}_{2}\left(\mathrm{a}^{1} \Delta_{g}\right)$ and $\mathrm{O}\left({ }^{1} \mathrm{D}\right)$ will be present in relatively small quantities (Park 1989; Klopovskii et al. 1995; Starik et al. 2009). However, the ground-state species are the primary product of ozone shock dissociation. In terms of excited species, the resulting singlet $\mathrm{O}\left({ }^{1} \mathrm{D}\right)$ is rapidly quenched (collisionally de-excited) by collisions with the ambient molecules, atoms and electrons (e.g. $\mathrm{N}_{2}, \mathrm{O}_{2}, \mathrm{O}$ ) and subsequently consumed by $\mathrm{N}_{2}$ (Zipf 1969; Capitelli et al. 2000; Fridman 2008; Schunk \& Nagy 2009).

Metastable $\mathrm{O}_{2}\left(\mathrm{a}^{1} \Delta_{\mathrm{g}}\right)$ is relatively immune to quenching by a major atmospheric gas (Zipf 1969), and may react under favourable high temperatures with meteoric metallic ion such as $\mathrm{Fe}^{+}$and $\mathrm{Mg}^{+}$.

Consequently, the thermally driven reactions between meteor metal ion species $\mathrm{M}^{+}$and oxygen (remaining from shock dissociation of $\mathrm{O}_{3}$ and ambient $\mathrm{O}_{2}$ that survived the shock wave) will proceed in the boundary region of the hot meteor trail as long as there are favourable temperature regimes:

$\mathrm{M}^{+}+\mathrm{O}_{2} \rightarrow \mathrm{MO}^{+}+\mathrm{O}$

However, the Maxwell-Boltzmann distribution of shock modified species behind the shock wave (Cercignani 2000) indicates that the surviving quantities of the ambient molecular oxygen will also participate in the same thermally controlled reaction, albeit towards the outer boundary of the $R_{0}$ region (our modelling results confirm this, see Section 4.3). However, in terms of the overall contribution to the reaction (9), oxygen from shock dissociated ozone may not play a dominant role within $R_{0}$, because the $\mathrm{O}_{3}$ concentration in the MLT is five orders of magnitude lower than that of $\mathrm{O}_{2}$.

In principle, reaction (9) is endothermic and will proceed rapidly in the hot meteor boundary region. When $\mathrm{M}=\mathrm{Fe}^{+}$or $\mathrm{Mg}^{+}$in the equations above, observational evidence indicates that under hyperthermal conditions, subsequent reactions proceed at the collisional 
rate (Ferguson \& Fehsenfeld 1968). Furthermore, the rate of the reaction depends on thermodynamic and mixing considerations in the boundary region between the shock modified ambient atmosphere and the metal ions produced in the ambipolarly diffusing meteor trail (Dressler 2001; Jenniskens et al. 2004; Berezhnoy \& Borovička 2010).

The formation of $\mathrm{MO}^{+}$will take place between $3000 \mathrm{~K}$ and $1500 \mathrm{~K}$ (for additional discussion on metal oxide formation in meteor trains at temperatures between 1500 and $4000 \mathrm{~K}$, see Berezhnoy \& Borovička 2010), which indicate a reasonable range of values of temperature in the meteor train boundary for the first $0.1 \mathrm{~s}$ (during the initial stage of the ambipolar expansion; Jenniskens et al. 2004). The process of production of a metal oxide ion by the hyperthermal reaction between $\mathrm{M}^{+}$and $\mathrm{O}_{2}$ yields highest quantities at about $2500 \mathrm{~K}$ and it is not appreciable below $800 \mathrm{~K}$, which is the value below which oxygen is inert (Zel'dovich \& Raizer 2002). In the lower temperature range, this process will cease to be relevant as the source of the metal ion oxides. Observational data of meteor wake temperatures and train thermalization (e.g. Jenniskens et al. 2004) supports our assertion that the metal oxide ion formation takes place following the adiabatic overdense meteor train 'instantaneous' expansion.

However, the thermochemistry of these reactions is poorly established (Dalleska \& Armentrout 1994), especially under MLT conditions. Armentrout, Halle \& Beauchamp (1982) investigated the reactions of $\mathrm{Cr}^{+}, \mathrm{Mn}^{+}, \mathrm{Fe}^{+}, \mathrm{Co}^{+}$and $\mathrm{Ni}^{+}$with $\mathrm{O}_{2}$, which yield metal oxide ions and reported on the reaction cross-sections as a function of ion kinetic energy. A number of studies have been conducted in the past investigating the thermal reactions between metals, such as $\mathrm{Fe}, \mathrm{Mg}, \mathrm{Al}$ and $\mathrm{O}_{2}$ (e.g. Fontijn et al. 1972; Fontijn \& Kurzius 1972). However, only a relatively small number of studies considering the metal ion reactions with oxygen have been performed (e.g. Armentrout et al. 1982).

Subsequently, the removal of electrons by the thermally formed meteor metal oxides (produced in the post-adiabatically diffusing train boundary) is an exothermic process that is both fast and temperature independent for meteor metal oxide ions (Plane et al. 2015):

$\mathrm{MO}^{+}+\mathrm{e}^{-} \rightarrow \mathrm{M}+\mathrm{O}$.

The time-scale of electron removal during this reaction depends on the number density of the newly formed $\mathrm{MO}^{+}$. Following the consumption of a critical number of meteor metal oxides, the reaction (10) will no longer be appreciable and then ambipolar diffusion takes over again (Fig. 1) as a dominant mechanism of electron removal from the meteor train. Depending on the altitude, the process of electron removal will be complete in approximately $0.1-0.3 \mathrm{~s}$. The above discussion indeed demonstrates that ozone (albeit indirectly) may play a role in a brief electron removal from the postadiabatically expanding meteor train boundaries. At the moment, in the absence of high-resolution numerical code that accounts for the shock induced chemical reactions of both major and minor MLT species in the rarefied atmosphere, we cannot estimate with certainty the ratios of shock dissociated ozone and ambient shock surviving $\mathrm{O}_{2}$ that participates in the initial thermally driven oxidation and subsequent post-hyperthermal dissociative recombination that removes electron from meteor train boundary (reaction 10; see the comments in Section 4.3). We can say that in the volume between $r_{0}$ and $R_{0}$, however, most $\mathrm{O}_{2}$ that originates from shock dissociation of $\mathrm{O}_{3}$ will be consumed by meteor metal ions. We can, however, estimate that the ratio of $\mathrm{O}_{2}$ that comes from ozone shock dissociation to the ambient $\mathrm{O}_{2}$ that participates in thermally driven oxidation of meteor metallic ion is in the range of $10^{-2}-10^{-3}$, depending on the axial distance from the meteor train (within $R_{0}$ ). This is still a considerable contribution from ozone, given that the ratio of $\mathrm{O}_{3}$ to $\mathrm{O}_{2}$ in the MLT is about $10^{-5}$.

In summary, it has been demonstrated that an initial hyperthermal chemical reaction (where the rate is governed by the temperature), which is subsequently followed by a dissociative recombination (that will primarily depend on the concentration and availability of metal oxide ions) may be instrumental in removing electrons from the post-adiabatically expanding high-temperature meteor trail. Moreover, both of these processes are competing against ambipolar diffusion. The best way to describe such a dynamic system is with the second Damköhler number ( $\mathrm{Da}_{\mathrm{II}}$ ) (Sarma 2000). Consequently, the electron removal in the boundary region of the ambipolarly expanding high-temperature meteor train will strongly depend on the second Damköhler number, which needs to be always considered when there are competing regimes of diffusion and chemical removal of electrons in the meteor train boundary region (Jakobsen 2008; Jarosinski \& Veyssiere 2009).

We note that $\mathrm{Da}_{\text {II }}$ is a function of ambipolar diffusion, temperature and species number density. For $\mathrm{Da}_{\mathrm{II}}>1$, the chemical removal of electrons dominates, while for $\mathrm{Da}_{\mathrm{II}}<1$, the ambipolar diffusion is a primary mechanism of electron removal.

Of course, the process of the electron removal discussed above may not be applicable farther away $\left(\geq R_{0}\right)$ from the boundary of the initially formed meteor train where the effects of the cylindrical shock waves attenuate rapidly. Specifically, we have shown in this section that the overdense meteor cylindrical shock wavedissociated ozone products [ground state $\mathrm{O}_{2}(\mathrm{X})$ and to a lesser degree $\mathrm{O}_{2}\left(\mathrm{a}^{1} \Delta_{\mathrm{g}}\right)$ ] play an important role through hyperthermal reactions with meteoric metal ions and subsequent dissociative recombination in electron removal from the boundary of the overdense meteor train in the early stages of the post-adiabatic trail expansion.

Interestingly, the species densities and evolution in the early stage of meteor trail boundary evolution, modelled by Zinn et al. (2004) and Zinn \& Drummond (2005), support our findings.

Finally, the importance of hyperthermal chemistry enabled by the relatively slow thermalization of the high-temperature meteor train (Jenniskens et al. 2004) and likely modification of the near-field region of the ambient atmosphere, even by a relatively weak shock wave, can be further extended not only to transitional but also to strong underdense meteors (e.g. see Lee et al. 2013 and Hocking et al. 2016).

\subsection{The computational model}

For illustrative purposes, we have modelled a simple hypersonic meteor flow without ablation in the MLT region. The broad aim was to emphasize the difference non-ablation makes relative to the ablating meteoroids, and make a qualitative comparison to those numerical models that do include ablation (e.g. Boyd 2000; Zinn et al. 2004). We applied a simplified model, incorporated into the computational fluid dynamics (CFD) software package ANSYS Fluent (http://www.ansys.com), to investigate the distribution and magnitude of the pressure and temperature fields behind the initial bow shock wave envelope, and to determine what fraction of $\mathrm{O}_{2}$ (if any) survives the initial meteor bow shock wave conditions. The computational model, along with the governing equations and rate parameters (based on Park 1989), is described in Niculescu et al. (2016). The code is optimized for simulating the formation and evolution of the bow shock wave in the continuum flow regime associated with the hypersonic flows and the model can resolve the chemical reactions of the major species in and behind the shock 


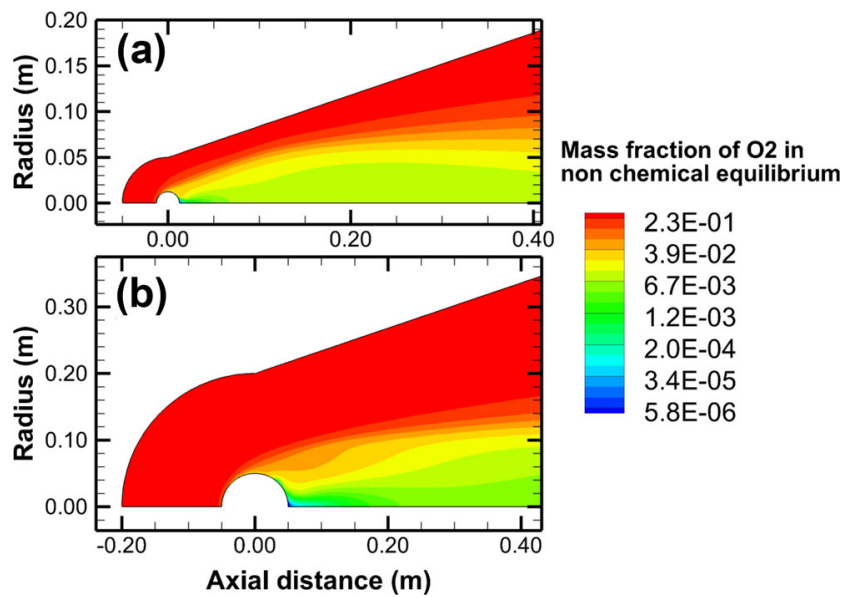

Figure 3. The mass fraction of $\mathrm{O}_{2}$ as a function of radial distance from the propagation axis of the (a) $2.5 \mathrm{~cm}$ and (b) $10 \mathrm{~cm}$ meteoroid. The top boundary ('white space' in the plot) represents a numerical boundary condition without any physical significance (it is set up to be far enough away from the body (meteoroid), such that the influence of the body (meteoroid) no longer has any effect. Note that (a) and (b) have different axes scaling. The colour scheme is represented in log scale.

wave (Niculescu et al. 2016). Shock waves, as well as the chemical reactions, including the dissociation of $\mathrm{N}_{2}$ and $\mathrm{O}_{2}$ are included in the model. However, the code does not currently include modelling certain minor species, such as $\mathrm{O}_{3}$. At the moment, this simple model is not optimized to resolve the effects of ablation, ionization and radiation. However, efforts are being made at the moment to incorporate those effects in future numerical simulations.

The computation was performed using $\mathrm{O}_{2}$ and $\mathrm{N}_{2}$ as the only major species, at an altitude of $80 \mathrm{~km}$. Relative to the ambient air, the initial mass fractions are $0.233\left(\mathrm{O}_{2}\right)$ and $0.767\left(\mathrm{~N}_{2}\right)$, and the initial molar fractions are $0.21\left(\mathrm{O}_{2}\right)$ and $0.79\left(\mathrm{~N}_{2}\right)$. A spherically shaped meteoroid is assumed to be moving at $35 \mathrm{~km} \mathrm{~s}^{-1}\left(M_{80 \mathrm{~km}}=124.6\right)$. The meteoroid diameters $\left(m_{\mathrm{d}}\right)$ considered in our simulations are $m_{\mathrm{d}}$ $=2.5 \mathrm{~cm}$ and $m_{\mathrm{d}}=10 \mathrm{~cm}$. The ratio of hydrodynamic-to-chemical time-scale of $\mathrm{O}_{2}$ formation and destruction for these two cases is 0.001 and 0.01 , respectively. Thus, we used the non-equilibrium approach.

The computational results representing the mass fraction of $\mathrm{O}_{2}$, pressure and temperature fields are shown in Figs 3-5. The radial temperature distribution is plotted in Fig. 6.

We have used the simulation results obtained here to infer the amount of ambient $\mathrm{O}_{2}$ that will survive the passage of the cylindrical shock wave (Fig. 3). Although the effect of dissociation on temperature in the flow field is included in the computational model, the present version of the code does not allow for a precise estimate of the amount of energy-dependent dissociation behind the cylindrical shock wave. Subsequent improvements in the code are needed to cover that aspect. Nevertheless, it is possible to infer with reasonable certainty that under the meteor cylindrical shock wave conditions (discussed in the main text, and Sections S1 and S2), not only will a significant amount of $\mathrm{O}_{2}$ survive, but some $\mathrm{O}_{2}$ that comes from $\mathrm{O}_{3}$ dissociation will also persist.

Since the effect of ablation is not included in the model, the magnitude of the pressure (Fig. 4) in the flow field is correspondingly smaller (e.g. Bronshten 1983). The recompression (cylindrical) shock wave can be seen forming and its magnitude and effects are negligible in comparison with the initial bow shock wave or the cylindrical shock wave in the case of ablation. In the absence of

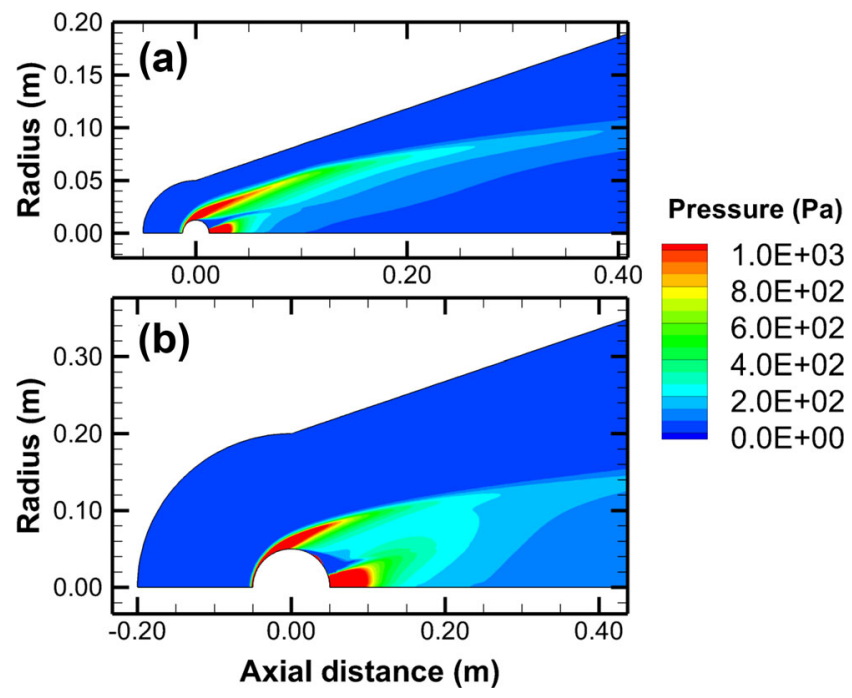

Figure 4. The pressure distribution around the propagation axis of the (a) $2.5 \mathrm{~cm}$ and (b) $10 \mathrm{~cm}$ meteoroid. The top boundary ('white space' in the plot) represents a numerical boundary condition without any physical significance [it is set up to be far enough away from the body (meteoroid), such that the influence of the body (meteoroid) no longer has any effect]. Note that (a) and (b) have different axes scaling.

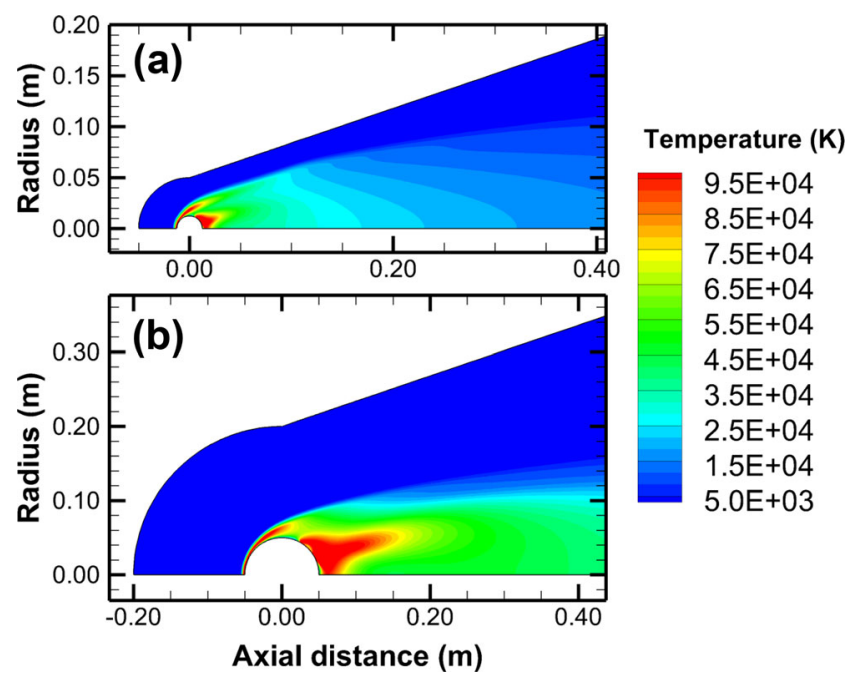

Figure 5. The temperature distribution around the (a) $2.5 \mathrm{~cm}$ and (b) 10 $\mathrm{cm}$ meteoroid. The top boundary (blank region in the plot) represents a numerical boundary condition without any physical significance [it is set up to be far enough away from the body (meteoroid), such that the influence of the body (meteoroid) no longer has any effect]. Note that (a) and (b) have different axes scaling.

ablation, the size of the flow field behind the initial shock region in front of the meteor is up to two orders of magnitude smaller than for the case of ablation (Boyd 2000; Jenniskens et al. 2000; Zinn et al. 2004). While there is a significantly smaller pressure increase behind the initial shock from the non-ablating spherical object, and overall reduced size of flow fields, the temperature magnitude (Fig. 5) remains reasonably similar to the case where ablation is considered (see Zinn et al. 2004 and Boyd 2000). The magnitude of the temperature (Fig. 6) is relatively similar to a scenario where strong ablation is present (Boyd 2000; Jenniskens et al. 2000; Zinn et al. 2004). However, the absence of the ablation will significantly reduce the magnitude of the radius of the volume around the me- 


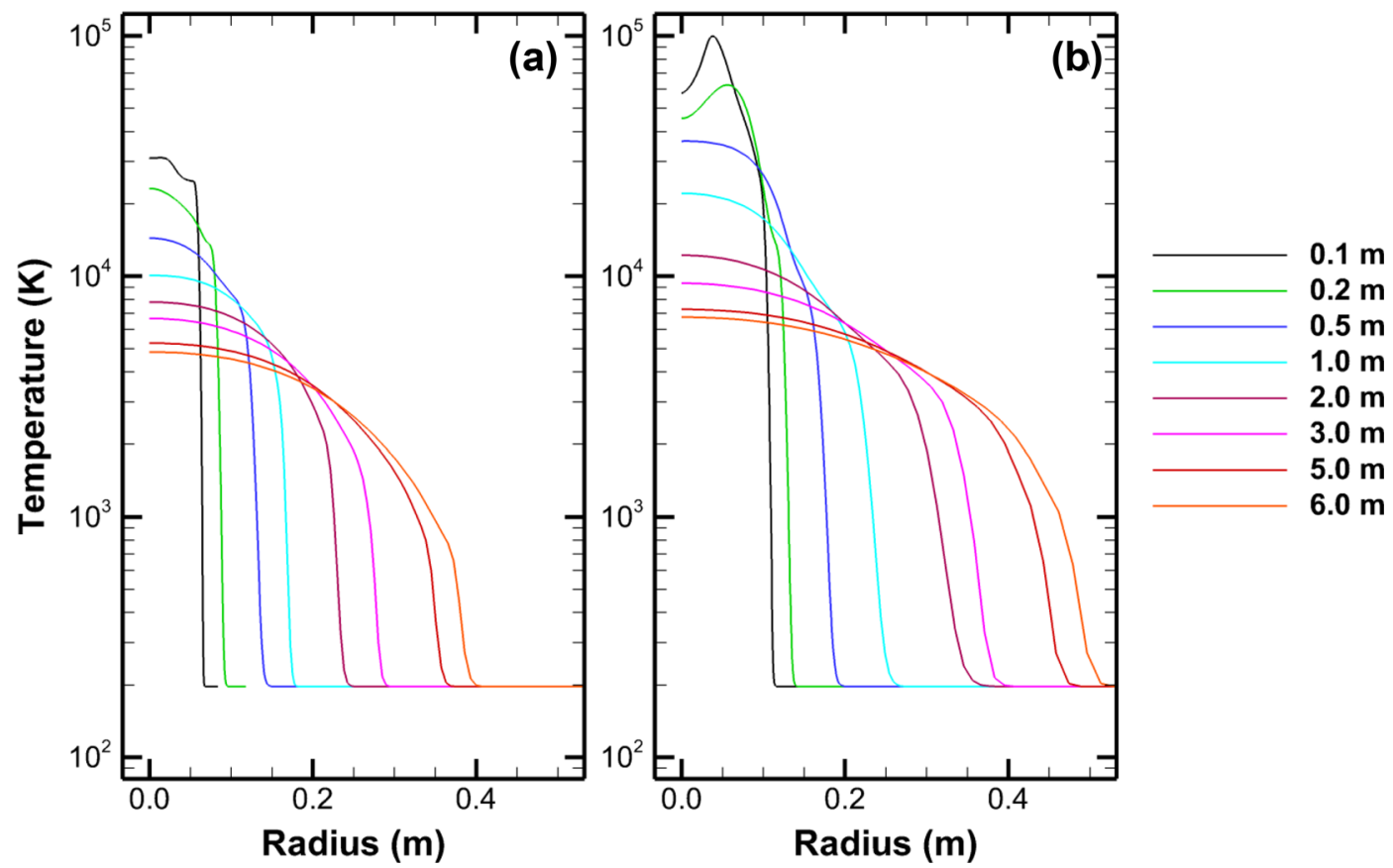

Figure 6. The radial temperature distribution as a function of distance from the (a) $2.5 \mathrm{~cm}$ and (b) $10 \mathrm{~cm}$ meteoroid (the vertical axis is in log scale). The legend represents the distance from the meteoroid along the propagation axis.

teor axis with an increased shock velocity dependent temperature. Although the effect of radiation is not included, our results (in spatially scaled down version) are consistent with those presented in Zinn et al (2004).

While the numerical model of the temperature and flow fields, along with the appearance of the bow shock wave, is in line with observations and other theoretical results (e.g. Viviani \& Pezzella 2015), we have demonstrated that in the absence of ablation, the flow regime remains unaffected, as predicted theoretically for the bodies of the specified sizes (Boyd 2000; Jenniskens et al. 2000; Zinn et al. 2004). Moreover, the recompression shock wave is substantially weaker than the radially expanding initial bow shock envelope, especially in comparison to the models that include ablation (Boyd 2000; Jenniskens et al. 2000) and observational results (Jenniskens et al. 2004). However, in the near field the radially expanding bow shock will still have modifying effects on the narrow region around the hypersonic body.

\section{SUMMARY AND CONCLUSIONS}

In this work, we have examined and presented the link between overdense meteor-generated shock waves and the short-lasting hyperthermal chemistry regime during the initial evolution of the meteor train. From the theoretical considerations, our results and conclusions can be summarized as follows.

I. Ablationally amplified cylindrical shock waves (approximated as blast waves from an explosive line source) produced by overdense meteors are strong enough to substantially modify the ambient atmosphere in the region near the initial point of maximum energy deposition per unit path length. The average overdense meteor cylindrical shock wave (which directly depends on pressure) heats the ambient atmosphere to about $6000 \mathrm{~K}$ in the near-field region $\left(<R_{0}\right)$. This theoretical calculation is based on determinations of both (i) the meteor velocity and delivered energy and (ii) the pressure ratio between the ablated and entrained vapour and plasma in front of the propagating meteoroid relative to the ambient atmosphere pressure. A temperature in the range of $6000 \mathrm{~K}$ is sufficient to dissociate both ozone and oxygen.

II. Specifically, we have demonstrated that in the range of initial temperatures in the region behind the strong overdense meteor cylindrical shock wave and in the meteor trail near-field (within $R_{0}$ ), large quantities of $\mathrm{O}_{2}$ will be dissociated. On the other hand, large quantities of both ground level and to a lesser extent, excited $\mathrm{O}_{2}$, which originate from ozone shock dissociation, survive. This is primarily due to the finite energy budget and short time-scales in the meteor region of MLT. However, both shock surviving ambient oxygen and that originating from $\mathrm{O}_{3}$ dissociation, hyperthermally react with the meteoric metal ions in the boundary region of the high temperature post-adiabatically expanding overdense meteor trail. The time-scales for high-temperature oxidation of meteoric metal ions depend strongly on the temperatures at the meteor boundary and altitude and are typically in the order of approximately $10^{-3} \mathrm{~s}$ at $80 \mathrm{~km}$.

III. Furthermore, for the case of overdense meteor trains, we have demonstrated that the subsequently formed meteoric metal oxide ions are predominantly responsible for the initial intense and short lasting electron removal from the boundary of the expanding meteor train, through a process of fast temperature-independent dissociative recombination. This height-dependent process is generally completed within $0.1-0.3 \mathrm{~s}$, which agrees well with the results indicating significantly slower cooling of meteor wakes (Jenniskens et al. 2004). The rate of this process is also strongly dependent on the second Damköhler number.

Finally, the findings presented in this paper are significant, as they illuminate the combined role of previously neglected effects of meteor-generated shock waves and hyperthermal chemistry in the role of radar-observed early diffusion of electrons in the meteor train boundary, which are consumed in the post-hyperthermal dissociative recombination. Evidently, there is a need for further 
validation using not only more capable numerical models but also additional observational and experimental studies.

\section{ACKNOWLEDGEMENTS}

EAS gratefully acknowledges the Natural Sciences and Engineering Research Council of Canada (NSERC) Postdoctoral Fellowship programme for partly funding this project. WKH acknowledges a Discovery Grant from the Natural Sciences and Engineering Research Council of Canada. MG acknowledges support from the ERC Advanced grant no. 320773, the Russian Foundation for Basic Research, project nos 16-05-00004 and 16-07-01072 and the Russian Science Foundation, project no. 14-22-00197. RES thanks Natural Sciences and Engineering Research Council of Canada Collaborative Research and Training Experience (NSERC CREATE) Program for Integrating Atmospheric Chemistry and Physics from Earth to Space (IACPES), and a Northern Scientific Training Program grant. Research at the Ural Federal University is supported by the Act 211 of the Government of the Russian Federation, agreement no. 02.A03.21.0006.

\section{REFERENCES}

Anderson J. D., 2006, Hypersonic and High Temperature Gas Dynamics. AIAA, VA

Armentrout P. B., Halle L., Beauchamp J., 1982, J. Chem. Phys., 76, 2449

Baggaley W., 1978, Planet. Space Sci., 26, 979

Baggaley W., 1979, Bull. Astr. Inst. Czechosl., 30, 184

Baggaley W., 1980, Symp. Int. Astronomical Union, Meteors and Atmospheres. Cambridge Univ. Press, Cambridge, p. 85

Baggaley W., Cummack C., 1974, J. Atmos. Sol.-Terr. Phys., 36, 1759

Baggaley W., Fisher G., 1980, Planet Space. Sci., 28, 575

Bauer E., 1990, Physics of High Temperature Air I, II. Institute for Defense Analyses, Alexandria, VA

Bennett F. D., 1958, Phys. Fluids, 1, 347

Benson S. W., Axworthy A. E., 1965, J. Chem. Phys., 42, 2614

Berezhnoy A. A., Borovička J., 2010, Icarus, 210, 150

Boyd I. D., 2000, in Jenniskens P., Rietmeijer F. J. M., Brosch N., Fonda M., eds, Leonid Storm Research. Springer, Netherlands, p. 93

Boyd I. D., Candler G. V., Levin D. A., 1995, Phys. Fluids, 7, 1757

Bronshten V. A., 1965, Problems of the Movements of Large Meteoric Bodies in the Atmosphere. National Aeronautics and Space Administration, USA

Bronshten V. A., 1983, Book Physics of Meteoric Phenomena. D. Reidel Publishing Co., Dordrecht

Brown P., Edwards W., ReVelle D., Spurny P., 2007, J. Atmos. Terr. Phys., 69,600

Brun R., 2009, Introduction to Reactive Gas Dynamics. Oxford Univ. Press, NY

Brun R., 2012, High Temperature Phenomena in Shock Waves. Springer, Berlin

Campbell-Brown M., Koschny D., 2004, A\&A, 418, 751

Capitelli M., Ferreira C. M., Gordiets B. F., Osipov A. I., 2000, Plasma Kinetics in Atmospheric Gases. Springer Science \& Business Media, NY, USA

Center R., Kung R., 1975, J. Chem. Phys., 62, 802

Ceplecha Z., Borovička J., Elford W. G., ReVelle D. O., Hawkes R. L., Porubčan V., Šimek M., 1998, Space Sci. Rev., 84, 327

Cercignani C., 2000, Rarefied Gas Dynamics: From Basic Concepts to Actual Calculations. Cambridge Univ. Press, Cambridge

Dalleska N., Armentrout P. B., 1994, Int. J. Mass Spectrom. Ion Process., 134, 203

Dobrovol'skii O. V., 1952, Byull. SAO, 6, 11

Dressler R. A., 2001, Chemical Dynamics in Extreme Environments. World Scientific, Singapore
Endo H., Glänzer K., Troe J., 1979, JPhCh, 83, 2083

Ferguson E., Fehsenfeld F., 1968, J. Geophys. Res., 73, 6215

Fontijn A., Kurzius S., 1972, CPL, 13, 507

Fontijn A., Kurzius S. C., Houghton J. J., 1972, DTIC Document. Princeton, NJ, p. 52

Francey J., 1963, AuJPh, 16, 500

Fridman A., 2008, Plasma Chemistry. Cambridge Univ. Press, Cambridge

Gidaspov V. Y., Losev S., Severina N., 2010, Math. Models Comput. Simul., 2,211

Gritsevich M., Koschny D., 2011, Icarus, 212, 877

Gritsevich M. I., 2008, Doklady Physics. Springer, Berlin, 53, 97-02

Gritsevich M. I., 2009, Adv. Space Res., 44, 323

Hayes W., Probstein R. F., 1959, Hypersonic Flow Theory. Academic Press, NY

Hocking W. K., Thayaparan T., Jones J., 1997, GeoRL, 24, 2977

Hocking W. K., Silber R. E., Plane J. M., Feng W., Garbanzo-Salas M., 2016, AnGeo, 34, 1119

Hurle I., 1967, Rep. Prog. Phys., 30, 149

Hutchens G. J., 1995, J. Appl. Phys., 77, 2912

Ibraguimova L. B., Shatalov O. P., Tunik Y. V., Zabelinskii I. E., 2012, in Kontis K., ed., 28th Int. Symp. Shock Waves: Vol. 1. Springer, Berlin, p. 125

Jakobsen H. A., 2008, Chemical Reactor Modeling. Springer, Berlin

Jarosinski J., Veyssiere B., 2009, Combustion Phenomena: Selected Mechanisms of Flame Formation, Propagation and Extinction. CRC Press, USA

Jenniskens P., 2004, Adv. Space Res., 33, 1444

Jenniskens P., Wilson M. A., Packan D., Laux C. O., Krüger C. H., Boyd I. D., Popova O. P., Fonda M., 2000, in Jenniskens P., Rietmeijer F. J. M., Brosch N., Fonda M., eds, Leonid Storm Research. Springer, NY, p. 57

Jenniskens, P., Laux, C. O., Wilson, M. A., Schaller, E. L., 2004, AsBio, 4,81

Johnston H. S., 1968, DTIC Document. US Dept. Commerce, Washington, DC, p. 58

Jones, J., Campbell-Brown, M., 2005, MNRAS, 359, 1131

Jones, W., Jones, J., 1990, J. Atmos. Sol.-Terr. Phys., 52, 185

Jones D., Goyer G., Plooster M., 1968, J. Geophys. Res., 73, 3121

Jones W., 1995, MNRAS, 275, 812

Jones W., 1997, MNRAS, 288, 995

Jones W., Halliday I., 2001, MNRAS, 320, 417

Jones W. M., Davidson N., 1962, J. Am. Chem. Soc., 84, 2868

Josyula E., Burt J., 2011, DTIC Document RTO-EN-AVT-194. NATO S\&T, USA, p. 40

Kaiser T., Greenhow J., 1953, Proc. Phys. Soc. B, 66, 150

Klopovskii K., Kovalev A., Lopaev D., Popov N., Rakhimov A., Rakhimova T., 1995, JETP, 80, 603

Kogan M., 1969, Rarefied Gas Dynamics. Plenum Press, NY

Konnov A., 2012, Energy Fuels, 27, 501

Lee C., Younger J., Reid I., Kim Y., Kim J. H., 2013, J. Geophys. Res.: Atmos., 118, 3037

Lees L., Hromas L., 1962, AIAA, 29, 976

Lin S. C., 1954, JAP, 25, 54

Lin S. C., Teare J. D., 1963, Phys. Fluids, 6, 355

Luther K., Oum K., Troe J., 2005, Phys. Chem. Chem. Phys., 7, 2764

McKinley D. W. R., 1961, Meteor Science and Engineering. McGraw-Hill, NY

McNeil W. J., Lai S. T., Murad E., 1998, J. Geophys. Res., 103, 10899

Makarov V., Shatalov O., 1994, FlDy, 29, 854

Massey H., Bates D., 1982. Applied Atomic Collision Physics. Academic Press, NY

Menees G. P., Park C., 1976, AtmEn, 10, 535

Michael J., 1971, J. Chem. Phys., 54, 4455

Murad E., 1978, J. Geophys. Res.: Atmos., 83, 5525

Nelson W. C., 1964, The High Temperature Aspects of Hypersonic Flow: Proceedings. Macmillan, USA

Nicolet M., Mange P., 1954, JGR, 59, 15

Niculescu M. L., Fadgyas M. C., Cojocaru M. G., Pricop M. V., Stoican M. G., Pepelea D., 2016, 4th Int. Workshop on Numerical Modelling in 
Aerospace Sciences, NMAS 2016. INCAS Bulletin, Bucharest, Romania, p. 55

Öpik E. J., 1958, Physics of Meteor Flight in the Atmosphere. Dover Publications, USA

Panesi M., Magin T., Huo W., Levin D. A., Wysong I. J., Garcia A. L., 2011, AIP Conf. Proc., 1333, 1251

Park C., 1989, 24th Thermophysics Conf. American Institute of Aeronautics and Astronautics, Buffalo, NY, p. 1740

Park C., Menees G. P., 1978, JGRC, 83, 4029

Peukert S., Sivaramakrishnan R., Michael J., 2013, JPCA, 117, 3729

Pickering W., Windle D., 1970, Planet. Space Sci., 18, 1153

Plane J. M., 2012, ChSRv, 41, 6507

Plane J. M., Feng W., Dawkins E. C., 2015, Chem. Rev., 115, 4497

Plooster M. N., 1968, Shock Waves from Line Sources, National Center for Atmospheric Research, Boulder, CO, p. 96

Plooster M. N., 1970, Phys. Fluids, 13, 2665

Popova O. P., Sidneva S. N., Shuvalov V. V., Strelkov A. S., 2000, Earth Moon Planets, 82, 109

Popova O., Sidneva S., Strelkov A., Shuvalov V., 2001, in Warmbein B., ed., ESA SP-495, Meteoroids 2001 Conf. ESA, Noordwijk, p. 237

Poulter E., Baggaley W., 1978, Planet. Space Sci., 26, 969

Poulter E., Baggaley W., 1977, J. Atmos. Sol.-Terr. Phys., 39, 757

Probstein R. F., 1961, ARS J., 31, 185

Räbinä J., Mönkölä S., Rossi T., Markkanen J., Gritsevich M., Muinonen K., 2016, JQSRT, 178, 295

Rajchl J., 1969, Bull. Astr. Inst. Czechosl., 20, 363

Rees M. H., 1989, Physics and Chemistry of the Upper Atmosphere. Cambridge Univ. Press, Cambridge

ReVelle D. O., 1974, PhD thesis, Michigan Univ.

ReVelle D. O., 1976, JGR, 81, 1217

Rogers L., Hill K., Hawkes R., 2005, Planet. Space Sci., 53, 1341

Romig M. F., 1964, The RAND Corporation (declassified). DTIC Document, Santa Monica, CA

Sarma G., 2000, PrAeS, 36, 281

Schumacher H., 1960, J. Chem. Phys., 33, 938

Schunk R., Nagy A., 2009, Ionospheres: Physics, Plasma Physics, and Chemistry. Cambridge Univ. Press, Cambridge

Shen C., 2006, Rarefied Gas Dynamics: Fundamentals, Simulations and Micro Flows. Springer, Berlin

Silber E. A., 2014, PhD Thesis, Western University

Silber E. A., Brown P. G., 2014, J. Atmos. Terr. Phys., 119, 116

Silber E. A., Brown P. G., Krzeminski Z., 2015, JGRE, 120, 413

Starik A., Titova N., Arsentiev I., 2009, Plasma Sources Sci. Technol., 19, 015007
Steiner H., Gretler W., 1994, Phys. Fluids, 6, 2154

Sugar G. R., 1964, Proc. IEEE, 52, 116

Takayama K., 2012, Shock Waves: Proceedings of the 18th Int. Symp. Shock Waves. Springer, Sendai, Japan

Tsikulin M., 1970. DTIC Document AD 715-537. Nat. Tech. Inform. Serv., Springfield

Viviani A., Pezzella G., 2015, Aerodynamic and Aerothermodynamic Analysis of Space Mission Vehicles. Springer, USA

Vondrak T., Plane J., Broadley S., Janches D., 2008, Atmos. Chem. Phys., 8,7015

Weryk R. J., Brown P. G., 2012, Planet. Space Sci., 62, 132

Weryk R. J., Brown P. G., 2013, Planet. Space Sci., 81, 32

William J., Murad E., 2002, in Murad E., Williams I. P., eds, Meteors in the Earth's Atmosphere: Meteoroids and Cosmic Dust and their Interactions with the Earth's Upper Atmosphere. Cambridge Univ. Press, Cambridge, p. 265

Yankovsky V., Manuilova R., 2006, AnGeo. Copernicus GmbH, 24, 2823

Zabelinskii I., Ibraguimova L., Shatalov O., Tunik Y. V., 2012, in Reijasse Ph., Knight D. D., Ivanov M. S., Lipatov I. I., eds, Progress in Flight Physics. EDP Sciences, France, p. 231

Zel'dovich Y. B., Raizer Y. P., 2002, Physics of Shock Waves and Hightemperature Hydrodynamic Phenomena. Dover Publications, Mineola, NY

Zinn J., Drummond J., 2005, J. Geophys. Res.: Space Phys., 110, A04306

Zinn J., O'Dean P. J., ReVelle D. O., 2004, Adv. Space Res., 33, 1466

Zipf E., 1969, Can. J. Chem., 47, 1863

\section{SUPPORTING INFORMATION}

Supplementary data are available at MNRAS online.

\section{meteor_shock_waves_supplement.pdf}

Please note: Oxford University Press is not responsible for the content or functionality of any supporting materials supplied by the authors. Any queries (other than missing material) should be directed to the corresponding author for the article.

This paper has been typeset from a $\mathrm{T}_{\mathrm{E}} \mathrm{X} / \mathrm{L} \mathrm{T} \mathrm{E} X$ file prepared by the author. 